\title{
Application of Data Compression and Decompression Based on Artificial Neural Network and Collaborative Computer Network
}

\author{
Li Dong, Binpeng Qu, Meng Zhang, Jing Yu \\ Shandong Medical College,Jinan,Shandong 250002, China \\ huner2011@foxmail.com
}

Keywords: Artificial neural network, Collaborative computer, Data compression.

\begin{abstract}
Before data transmission, in order to improve the utilization ratio of transmission channel, there is need to compress the transmitted data. Before data storage, in order to save storage space, there is need to compress the stored data. There are many methods for data compression and decompression, but the existing methods may be not effective for some particular applications. Therefore, for the special applications, special compression and decompression methods should be applied, which means to combine neural network and collaboration cognitive model. When finite multi-dimensional data needs to transmit with long distance or store data for a long time, the method combining neural network and collaborative computer should be applied to implement compression and decompression of finite multi-dimensional data.
\end{abstract}

\section{Introduction}

Encoding and decoding is a typical data compression method without considering the conditions of the compressed objects, and it only adopts a fixed encoding way. And the data compression ratio has tended to be saturated (10:1). On the other hand, selective power of information is deficient, so lots of data without relating to tasks in original data is reserved, which makes important information loss.

Traditional methods have the above limitations, so using artificial neural network for data compression has attracted the attention of the people. Compared with the traditional compression methods, the method has the advantages of good fault tolerance, self-organization and adaptivity. Therefore, in data compression, there is no need to use the predetermined data coding way. And the data is encoded and decompressed according to the characteristics of precompressed data.

However, applying artificial neural network for data compression and decompression has disadvantages. With the improvement of compression, training time is less and less, but the error increases. For decompressing the finite multi-dimensional data based on collaborative computer network, only accurately recognizing the prototype of compression data can realize lossless decompression, which is better than the artificial neural network.

\section{Basic Principles of BP Neural Network Data Compression}

Applying a three-tier BP network for data compression can make multi-dimensional data input have low-dimensional data output, which achieves the objective of data compression.

Firstly, a three-tier BP network is structured for data compression. Then, the link weight between input layer and middle layer and between middle layer and output layer is determined, and the link weight is modified by the error of actual output and expected output until it meets the given error. And the trained network can ensure that according to BP neural network output decision rule (when the output of a output node is greater than 0.9 , the output of the output node is 1 . When the output of a output node is 0.9 , the output node is 0 ), the output approximate to a binary number.

The compression of 10 12-dimensional data is taken as an example, and three-tier BP network is used to compress the data. There are 10 data to be compressed, so 4-bit binary number is used to express 10 different data. The input node of BP network is 12,12-dimensional data. There are 4 output nodes, 4-bit binary number. The ratio between the bits of network inputting 12-dimensional data and the number of outputting neuron nodes is the data compression ratio, which can refer to the 
formula $m=\log _{2} n$ (n is the input neurons)to design the nodes of hidden layer. A 12-dimensional data to be compressed is used as the input of network, and there is the network output. Properly processing a 12-dimensional data can get an only four-bit binary code. After compressing a 12-dimensional data, a four-bit binary number ban be used to express, which makes the original data compressed.

\section{Implementation of BP Neural Network Data Compression}

10 12-dimensional data to be compressed is taken as an example, and the data in Table 1 is the original data to be compressed.

Table 1 Original data to be compressed

\begin{tabular}{|c|c|c|c|c|c|c|c|c|c|c|c|c|}
\hline \multirow{2}{*}{ No. } & \multicolumn{10}{|c|}{ Data compression input data } \\
\cline { 2 - 14 } & $x_{1}$ & $x_{2}$ & $x_{3}$ & $x_{4}$ & $x_{5}$ & $x_{6}$ & $x_{7}$ & $x_{8}$ & $x_{9}$ & $x_{10}$ & $x_{11}$ & $x_{12}$ \\
\hline 1 & 0.12 & 0.19 & 0.22 & -0.44 & 0.79 & 0.92 & 0.87 & -0.90 & 0.27 & 0.16 & -0.42 & 0.24 \\
\hline 2 & 0.25 & 0.44 & -0.49 & 0.95 & 0.36 & -0.42 & 0.93 & 0.34 & 0.95 & 0.76 & 0.70 & 0.42 \\
\hline 3 & -0.95 & 0.88 & 0.96 & -0.18 & -0.25 & 0.14 & -0.69 & 0.59 & 0.55 & 0.48 & -0.85 & 0.99 \\
\hline 4 & 0.55 & 0.62 & 0.72 & -0.28 & 0.18 & 0.54 & 0.85 & -0.26 & 0.75 & 0.94 & -0.08 & 0.58 \\
\hline 5 & 0.98 & -0.76 & 0.54 & 0.16 & -0.19 & 0.60 & -0.96 & 0.84 & 0.64 & -0.43 & 0.25 & 0.27 \\
\hline 6 & 0.00 & 0.24 & -0.18 & 0.55 & 0.49 & 0.40 & 0.29 & -0.48 & 0.47 & 0.58 & 0.88 & -0.45 \\
\hline 7 & -0.02 & -0.25 & 0.38 & 0.66 & -0.75 & -0.93 & 0.18 & 0.25 & -0.45 & 0.12 & 0.20 & 0.96 \\
\hline 8 & -0.46 & 0.10 & -0.35 & 0.32 & 0.39 & 0.48 & -0.36 & 0.65 & 0.75 & 0.74 & -0.60 & 0.96 \\
\hline 9 & 0.52 & -0.65 & 0.74 & 0.91 & -0.62 & 0.99 & 0.62 & -0.82 & 0.40 & 0.20 & 0.72 & -0.52 \\
\hline 10 & 0.68 & -0.28 & -0.76 & 0.16 & 0.32 & -0.42 & 0.38 & 0.46 & 0.00 & -0.21 & 0.32 & 0.10 \\
\hline
\end{tabular}

BP neural network output decision rule is that when the output of an output node is greater than 0.9 , the output of the output node is 1 , when the output of an output node is less than 0.9 , the output node is 0 .

If 12-dimensional data in Number 8 of the above table $(-0.46,0.10,-0.35,0.32,0.39$, 0.48,-0.36,0.65,0.75,0.74,-0.60,0.96) is input BP network for compression, the operation result of the output side is $(0.9704,0.0004,0.0226,0.0188)$. According to BP neural network output decision rule, the binary code output is 1000 , which means that after data compression, 12-dimensional data of the line is changed to be a four-bit binary code 1000, and so on.

Three-tier BP network is used to compress the experimental data in Table 1, and according to BP neural network output decision rule, we can get the result in Table 2.

Table 2 Output value of BP compression network and binary codes

\begin{tabular}{|c|c|c|c|c|c|c|c|c|}
\hline \multirow{2}{*}{ No. } & \multicolumn{4}{|c|}{ Output of data compression network } & \multicolumn{4}{c|}{ Output of binary codes } \\
\cline { 5 - 9 } & \multicolumn{3}{|c|}{$\mathbf{n n n y y y y}_{1}$} & $c_{1}$ & $c_{2}$ & $c_{3}$ & $c_{4}$ \\
\hline 1 & 0.0298 & 0.0250 & 0.0013 & 0.9731 & 0 & 0 & 0 & 1 \\
\hline 2 & 0.0295 & 0.0340 & 0.9970 & 0.0002 & 0 & 0 & 1 & 0 \\
\hline 3 & 0.0144 & 0.0273 & 0.9751 & 0.9741 & 0 & 0 & 1 & 1 \\
\hline 4 & 0.0027 & 0.9701 & 0.0213 & 0.0237 & 0 & 1 & 0 & 0 \\
\hline 5 & 0.0256 & 0.9725 & 0.0199 & 0.9821 & 0 & 1 & 0 & 1 \\
\hline 6 & 0.0028 & 0.9675 & 0.9810 & 0.0297 & 0 & 1 & 1 & 0 \\
\hline 7 & 0.0052 & 0.9720 & 0.9981 & 0.9778 & 0 & 1 & 1 & 1 \\
\hline 8 & 0.9704 & 0.0004 & 0.0226 & 0.0188 & 1 & 0 & 0 & 0 \\
\hline 9 & 0.9684 & 0.0313 & 0.0027 & 0.9915 & 1 & 0 & 0 & 1 \\
\hline 10 & 0.9717 & 0.0226 & 0.9765 & 0.0093 & 1 & 0 & 1 & 0 \\
\hline
\end{tabular}

Therefore, we can get the following conclusion. Applying BP neural network for data compression can make a multi-dimensional data become low-dimensional data, which can achieve the objective of compression and is easy for storage or transmission. 


\section{Basic Principle and Implementation of Data Decompression Based on BP Network and Collaborative Computer Network}

Basic principles of data compression based on BP network and collaborative computer network. According to the characteristics of finite data compression and decompression, the research combines BP neural network and collaborative computer network to transform the structure of collaborative computer network. The order parameter layer and output layer of collaborative computer and the link structure of them is reserved to transform the link structure of input layer and the order parameter layer, and the output of BP decompression network is used as the input of collaborative computer network.

Data decompression network consists of left dotted box, decision generation order parameter unit and right dotted box. The left dotted box is a completed BP decompression network, and the right dotted box is the order parameter layer and output layer of collaborative computer network. The input neurons of BP network of left dotted box are 4, and the output neurons are 10 . There are 10 kinds of 12-dimensional data, so the number of order parameter nodes of collaborative computer network in right dotted box is 10 , and the number of output nodes is 12 .

4.2 Implementation of data decompression based on BP neural network and collaborative computer network

The data after compression in Table 2 is taken as an example. If the four-bit binary code (1000) input data of number 8 receives decompression, a 10-dimensional data $(0.0006,0.0006,0.0000$, $0.0155,0.0019,0.0001,0.0000,0.9722,0.0163,0.0244$ ) is output on BP network, the data generates order parameter unit decision, and generates a group of 10-dimensional order parameters $(0,0,0$, $0,0,0,0,1,0,0)$. The artithmetic expression of 10-dimensional order parameter by the weight of collaborative network order parameter layer and output layer is $X_{i}=\sum_{k=1}^{m} \xi_{k} v_{k j} \quad \mathrm{j}=1,2 \ldots, \mathrm{n}$; $\mathrm{k}=1,2 \ldots, \mathrm{m}$, which can get a group of 12 -dimensional original data $(-0.46,0.10,-0.35,0.32$, $0.39,0.48,-0.36,0.65,0.75,0.74,-0.60,0.96$ ) .

The data in Table 2 is the input of BP decompression network, which can get the following experimental results.

The final learning error is $\mathrm{e}=0.0100$.

Learning times is $\mathrm{T}=2584$.

The output decision rule of BP network can be used to get the input data of collaborative computer network, as shown in Table 3.

Table 3 Binary output value of BP decompression network

\begin{tabular}{|c|c|c|c|c|c|c|c|c|c|c|}
\hline No. & \multicolumn{10}{|c|}{ Output of BP decompression network } \\
\hline 1 & 1 & 0 & 0 & 0 & 0 & 0 & 0 & 0 & 0 & 0 \\
\hline 2 & 0 & 1 & 0 & 0 & 0 & 0 & 0 & 0 & 0 & 0 \\
\hline 3 & 0 & 0 & 1 & 0 & 0 & 0 & 0 & 0 & 0 & 0 \\
\hline 4 & 0 & 0 & 0 & 1 & 0 & 0 & 0 & 0 & 0 & 0 \\
\hline 5 & 0 & 0 & 0 & 0 & 1 & 0 & 0 & 0 & 0 & 0 \\
\hline 6 & 0 & 0 & 0 & 0 & 0 & 1 & 0 & 0 & 0 & 0 \\
\hline 7 & 0 & 0 & 0 & 0 & 0 & 0 & 1 & 0 & 0 & 0 \\
\hline 8 & 0 & 0 & 0 & 0 & 0 & 0 & 0 & 1 & 0 & 0 \\
\hline 9 & 0 & 0 & 0 & 0 & 0 & 0 & 0 & 0 & 1 & 0 \\
\hline 10 & 0 & 0 & 0 & 0 & 0 & 0 & 0 & 0 & 0 & 1 \\
\hline
\end{tabular}

Therefore, the method is used to decompress the data in Table 2. At the output end of collaborative computer network, the decompression results can be achieved, as shown in Table 4 . 
Table 4 Final decompression results of data decompression network

\begin{tabular}{|c|c|c|c|c|c|c|c|c|c|c|c|c|}
\hline \multirow{2}{*}{ No. } & \multicolumn{10}{|c|}{ Input data of data decompression network } \\
\cline { 2 - 14 } & $x_{1}$ & $x_{2}$ & $x_{3}$ & $x_{4}$ & $x_{5}$ & $x_{6}$ & $x_{7}$ & $x_{8}$ & $x_{9}$ & $x_{10}$ & $x_{11}$ & $x_{12}$ \\
\hline 1 & 0.12 & 0.19 & 0.22 & -0.44 & 0.79 & 0.92 & 0.87 & -0.90 & 0.27 & 0.16 & -0.42 & 0.24 \\
\hline 2 & 0.25 & 0.44 & -0.49 & 0.95 & 0.36 & -0.42 & 0.93 & 0.34 & 0.95 & 0.76 & 0.70 & 0.42 \\
\hline 3 & -0.95 & 0.88 & 0.96 & -0.18 & -0.25 & 0.14 & -0.69 & 0.59 & 0.55 & 0.48 & -0.85 & 0.99 \\
\hline 4 & 0.55 & 0.62 & 0.72 & -0.28 & 0.18 & 0.54 & 0.85 & -0.26 & 0.75 & 0.94 & -0.08 & 0.58 \\
\hline 5 & 0.98 & -0.76 & 0.54 & 0.16 & -0.19 & 0.60 & -0.96 & 0.84 & 0.64 & -0.43 & 0.25 & 0.27 \\
\hline 6 & 0.00 & 0.24 & -0.18 & 0.55 & 0.49 & 0.40 & 0.29 & -0.48 & 0.47 & 0.58 & 0.88 & -0.45 \\
\hline 7 & -0.02 & -0.25 & 0.38 & 0.66 & -0.75 & -0.93 & 0.18 & 0.25 & -0.45 & 0.12 & 0.20 & 0.96 \\
\hline 8 & -0.46 & 0.10 & -0.35 & 0.32 & 0.39 & 0.48 & -0.36 & 0.65 & 0.75 & 0.74 & -0.60 & 0.96 \\
\hline 9 & 0.52 & -0.65 & 0.74 & 0.91 & -0.62 & 0.99 & 0.62 & -0.82 & 0.40 & 0.20 & 0.72 & -0.52 \\
\hline 10 & 0.68 & -0.28 & -0.76 & 0.16 & 0.32 & -0.42 & 0.38 & 0.46 & 0.00 & -0.21 & 0.32 & 0.10 \\
\hline
\end{tabular}

The research results indicate that combining collaborative computer network and BP neural network can decompress finite multi-dimensional data, which can ensure that the data is lossless after decompression.

\section{Conclusions}

The paper combines the principles of BP neural network and collaborative computer network, and proposes and researches feasible data compression and decompression method for finite multi-dimensional data. According to the characteristics of finite multi-dimensional data compression and decompression, the paper establishes neural network for data compression of finite multi-dimensional data. And the paper combines neural network and collaborative computer to build a mixing network combining neural network and collaborative computer for data decompression of finite multi-dimensional data.

\section{References}

[1] H. Haken. Synergetic computers and recognition-a top-down approach to neural nets. Berlin:Springer-Verlag,1991

[2] Hu Dongliang, Qi Feihu , Liu Jianfeng. Recognition of objects with skew distortion based on synergetics. Pattern Recognition Letters 20(1999) 255-265

[3] Peter, Joerg; Freyer, Richard; Image segmentation with constraint satisfaction synergetic potential network Proc. SPIE Vol. 2424, p. 384-393, Nonlinear Image Processing VI, Edward R. Dougherty; Jaakko T. Astola; Harold G. Longbotham; Nasser M. Nasrabadi; Aggelos K. Katsaggelos; Eds. 03/1995

[4] R. W. Frischholz, F. G. B öbel, and K. P. Spinnler Face Recognition with the Synergetic Computer.International Conference on Applied Synergetics and Synergetic Engineering, Erlangen 1994.

[5] J. A. K. Suykens et. al. On-Line Learning Fokker-Plank Machine. Neural Processing Letters 7:81-89,1998

[6] H. Maeda et. al. A New Method for Matching Problem in Computer Vision Using Synergetics. 1999 IEEE International Conference on Systems, Man, and Cybernetics, October 12-15, 1999Tokyo, Japan: 503-508 